\title{
A SIMULTANEOUS TWO CHANNEL PHOTOMETRIC SYSTEM
}

\author{
D. J. Sullivan and A. van der Peet \\ Physics Department \\ Victoria University of Wellington \\ Wellington, NEW ZEALAND
}

ABSTRACT. A photometric system capable of simultaneous dual channel operation has been developed for use in NZ. The photometer head unit, developed and constructed by the University of Texas at Austin, permits simultaneous observation of two stars using suitable optics and mechanically coupled photomultiplier systems. The instrumentation and control system is centred around a DEC micro/PDP-11 computer and incorporates general purpose hardware and software developed specifically for the system.

\section{INTRODUCTION}

There is no doubt that a research programme for a small telescope should include photometry of variable stars. Monitoring stellar variability using ground-based telescopes is, of course, limited by atmospheric disturbances and, in particular the subtle changes in atmospheric transparency that sometimes accompany an otherwise good night. Simultaneous observation of a comparison star along with the star of interest is an obvious and now well established approach to this problem. A number of years observing experience with New Zeal and's island climate has convinced us that the two channel approach is a virtual necessity for accurate and reliable photometry, especially where relatively short integration times are involved. For our two channel system, we have chosen the option of using a divided light path and two photomultiplier systems. Another approach combines a single photomultiplier with a fast chopping method, enabling effective simultaneous observation of two stars for time scales long relative to the chopping period. This has the advantage of avoiding any differential drifts between two photomultiplier tubes, but photometric efficiency is halved. Although recent developments in high efficiency 2D detectors (such as the charge coupled devices) now make this a preferred option, the current high capital costs involved will not fit within our small telescope budget. 


\section{HARDWARE}

The Texas photometer-telescope unit employs a standard light path for the primary channel: light reaches the main photomultiplier via an aperture, a filter wheel driven by a stepper motor, and a fabry lens for imaging the telescope mirror on the photocathode. A 45 degree mirror containing a hole for the primary channel is positioned at the entrance to the photometer, and an adjustable 2D slide arrangement permits accurate location of a second stellar image in a narrow annulus in the field surrounding the primary object. This offset guiding mechanism can be used directly, or a flip mirror allows redirection of the light to a second smaller photomultiplier, via an aperture, stepping motor-controlled filter wheel and fabry lens.

Data acquisition and control for the system is accomplished using a DEC micro/PDP-11, featuring a 10 Mbyte hard disk and $5 \frac{1}{4}$ inch floppy disks. A number of special purpose boards have been constructed. Single photoelectron pulses from the photomultipliers are amplified and shaped using units similar to Taylor (Taylor, 1980), and these enter the computer environment via counter/buffer hardware on one interface board. The other interface unit features items for analogue display, stepper motor control and time synchronisation with an external crystal-controlled clock.

\section{SOFTWARE}

A general purpose software package for the computer system has been produced. It follows the framework of a package produced previously for another system (Sullivan, 1982), but incorporates new features provided by the micro/PDP-11. Control of, and responses to, external hardware are handled by the multiple priority interrupt system, while background routines perform a range of tasks that are not timecritical. An important component in the software package (as with any modern instrument) are routines which allow real-time display of the incomming data streams in a variety of ways. The software has been kept as general as possible in order to allow flexible development for a number of proposed programmes ranging from high-speed photometry to multiple-filter photometry of eclipsing binary stars.

\section{ACKNOWLEDGEMENTS}

The authors would like to acknowledge the generous financial support of the NZ Lottery Board and University Research Grant Committee.

\section{REFERENCES}

Sullivan, D.J. (1982), Southern Stars 30, 19

Taylor, D.J. (1980), Pub.Astr.Soc.Pac. 92, 108 